\title{
Deriving the absolute wave spectrum from an encountered distribution of wave energy spectral densities
}

\author{
Nielsen, Ulrik Dam
}

Published in:

Ocean Engineering

Link to article, DOI:

10.1016/j.oceaneng.2018.07.046

Publication date:

2018

Document Version

Peer reviewed version

Link back to DTU Orbit

Citation (APA):

Nielsen, U. D. (2018). Deriving the absolute wave spectrum from an encountered distribution of wave energy spectral densities. Ocean Engineering, 165, 194-208. https://doi.org/10.1016/j.oceaneng.2018.07.046

\section{General rights}

Copyright and moral rights for the publications made accessible in the public portal are retained by the authors and/or other copyright owners and it is a condition of accessing publications that users recognise and abide by the legal requirements associated with these rights.

- Users may download and print one copy of any publication from the public portal for the purpose of private study or research.

- You may not further distribute the material or use it for any profit-making activity or commercial gain

- You may freely distribute the URL identifying the publication in the public portal

If you believe that this document breaches copyright please contact us providing details, and we will remove access to the work immediately and investigate your claim 


\title{
Deriving the absolute wave spectrum from an encountered distribution of wave energy spectral densities
}

\author{
Ulrik D. Nielsen ${ }^{\mathrm{a}, \mathrm{b}}$ \\ ${ }^{a}$ DTU Mechanical Engineering, Technical University of Denmark, DK-2800 Kgs. Lyngby, Denmark \\ ${ }^{b}$ Centre for Autonomous Marine Operations, NTNU AMOS, NO-7491 Trondheim, Norway
}

\begin{abstract}
The objective of ship motion-based wave spectrum estimation is to provide the distribution of wave energy densities in absolute domain. However, as a ship generally advances relative to the progressing waves, any spectrum estimate inherently dates back to the encounter domain and, consequently, the spectrum estimate must be transformed to absolute domain. In following sea conditions, spectrum transformation from encounter to absolute domain has no unique (mathematical) solution. This article presents an optimisation-based technique to carry out the particular transformation in following sea conditions. The optimisation relies on an object function established using (wave) spectral moments; calculated directly using the estimated encounter-wave spectrum on the one side and by using a parameterised wave spectrum valid in absolute domain on the other side. The simplicity of the transformation technique is a strength in itself as it leads to an insignificant computational effort in the transformation to absolute domain. Equally important, the specific technique proves capable to provide accurate results in the majority of cases, when comprehensive testings with numerically simulated data of following sea conditions are performed. Furthermore, the technique is tested successfully using experimental full-scale sea trials data.
\end{abstract}

Keywords:

Ocean wave spectrum, advancing ship, Doppler Shift, encounter and absolute domains, spectrum transformation, following waves

Email address: udn@mek.dtu.dk (Ulrik D. Nielsen) 


\section{Introduction}

When a ship advances in its seaway (on deep water), the relative velocity and the angle between vessel and the progressing waves are the parameters that determine the observed period of the encountered waves. Mathematically, this phenomenon is described by the Doppler Shift which expresses that the encountered wave frequency, observed on a ship advancing relative to the inertial frame of reference, is different from the absolute wave frequency. Consequently, the Doppler Shift needs to be strictly taken into account when theoretical calculations of wave-induced responses are to be made since, otherwise, it is not possible to compare the result to any corresponding set of measurements. Note, in the remaining parts, deep-water conditions are assumed throughout. Moreover, all operational parameters, including vessel advance speed and encounter-wave angle, are constant.

It is a fact, see Section 2, that the Doppler Shift relates one - unique - absolute (wave) frequency to any one single encountered frequency when the velocity vectors of the ship and the waves have opposing projections on the ship's centreline, meaning that the angle between the pair of velocity vectors is larger than 90 degrees and smaller than 270 degrees; qualitatively expressed as the ship is in beam to head sea conditions. On the other hand, the Doppler Shift associates three absolute frequencies to any one single encountered frequency when two conditions are fulfilled, (1) the ship "follows" the progressive waves, (2) the encounter frequency takes a value less than a certain number, noting that the first condition implies that the angle between the pair of velocity vectors is either smaller than 90 degrees or larger than 270 degrees for the 1-to-3 relationship to be occurring.

The existing literature about the Doppler Shift, including its implications for ships sailing in waves, is fairly extensive when it comes to conceptual understanding and theoretical studies, see e.g. Lewandowski (2004); Price and Bishop (1974); Bhattacharyya (1978); Beck et al. (1989); Journée and Massie (2001). However, the situation is different when focus is on practical calculations related to real-case scenarios and applications. This is indeed so because of the 3-to-1 relationship, or mapping, between corresponding sets of frequencies in the absolute and the encounter domains when a ship advances in following waves*. Thus, any set of wave-induced measurements cannot be uniquely

*While it may be a 3 -to-1 mapping in the one "direction", it will be a 1-to-3 mapping in the other. 
Anyhow, the problem will generally from this point just be referred to as a 1-to-3 mapping problem. 
very efficient and capable in providing accurate estimates of the encounter-wave spectrum together with the relative wave heading angle for ships with advance speed. It is therefore highly relevant to address how to obtain the corresponding absolute wave energy spectrum.

In a previous work by Nielsen (2017), a practical approach was suggested for the transformation from encounter to absolute domain of wave spectral densities. The approach was tested comprehensively with numerical simulations, and the method has also been studied together with full-scale experimental data for its purpose in relation to ship motion-based wave spectrum estimation. Generally, the approach has been found to perform satisfactory but examples of the opposite also occur (Nielsen and Brodtkorb, 2018). The present work is introducing a second alternative transformation technique which relies on optimisation of a set of characteristic - absolute - wave parameters (e.g. significant wave height and zero-upcrossing period) based on an object function formulated through the wave spectral moments. This particular approach makes the transformed spectrum, in absolute domain, to have a shape of a parameterised wave spectrum such as a generalised JONSWAP spectrum (Hasselmann et al., 1973). It is the overall objective of this article to compare the new optimisation-based transformation technique with the "old" transformation technique (Nielsen, 2017).

Besides the introduction, the article consists of five sections: Details and consequences of the Doppler Shift set the problem faced when spectrum transformation from encounter to absolute domain must be conducted, and this is explained in Section 2. The remedy in terms of a (new) specific transformation algorithm is given in Section 3, while application and testings on data are outlined in Sections 4 and 5 which contain performance evaluations, including comparative studies between the old and the new transformation techniques. Finally, Section 6 summarises and brings the conclusions and suggestions for further work.

\section{Problem formulation}

The distribution of wave energy density $S(\omega)$, or simply the wave spectrum, in a fixed point is considered, where $\omega$ is the absolute frequency. The wave energy in the infinitesimal range $d \omega$ is proportional to $S(\omega) d \omega$ and, as a result, the total energy of 
The Doppler Shift implies that the wave encounter-frequency, $\omega_{e}$, is different from the absolute wave frequency $\omega_{0}$, and it can be shown that the mathematical relationship (on deep water) is given by,

$$
\omega_{e}=\omega_{0}-\omega_{0}^{2} \psi, \psi=\frac{U}{g} \cos \chi
$$

where $U$ is the speed of the vessel, and $\chi$ is the angle between the pair of velocity vectors of the vessel and the waves, respectively. Note that indices 0 (absolute) and (encounter) are used to emphasise the particular domains in question.

Although the vessel encounters waves at a frequency which is different from the absolute one, the total energy of the wave system is the same in the two domains, expressed as

$$
E\left(\omega_{e}\right) d \omega_{e}=S\left(\omega_{0}\right) d \omega_{0}
$$

where $E\left(\omega_{e}\right)$ refers to the encountered distribution of wave energy density, while $S\left(\omega_{0}\right)$ is the corresponding one in absolute domain. Consequently, wave energy spectral density can, in theory, be easily transformed from the one domain to the other, simply by multiplication with the derivative, $\frac{d \omega_{0}}{d \omega_{e}}$ or $\frac{d \omega_{e}}{d \omega_{0}}$, depending on the 'transformation direction';

$$
E\left(\omega_{e}\right)=S\left(\omega_{0}\right) \frac{d \omega_{0}}{d \omega_{e}}
$$

$$
S\left(\omega_{0}\right)=E\left(\omega_{e}\right) \frac{d \omega_{e}}{d \omega_{0}}
$$

As was indicated in the Introduction, wave spectrum transformation can be uniquely carried out when a ship sails "against" the waves (beam to head sea). In certain conditions related to following seas*, however, there exists no unique solution to the problem, since the Doppler Shift, Eq. (1), imposes a 1-to-3 relation between encounter frequency

*Onwards, "following waves" or "following seas" are used to indicate a situation in which the 1-to-3 mapping problem occurs, without having a specific relative wave heading in study. 
and absolute frequency, that is, one 'encountered wave component' is the result of three (true) absolute wave components. Consequently, the energy density at the specific encounter frequency cannot be assigned uniquely to the corresponding set of absolute frequencies as illustrated in Figure 1. This very problem has been comprehensively studied in Nielsen (2017), resulting in an elaborate, but at the same time practical, transformation algorithm. Briefly said, the algorithm is based on a scaling approach that assures preservation of energy (density) at corresponding sets of encounter and absolute frequencies by mapping the set of absolute frequencies into energy densities of a standard parameterised wave energy spectrum (e.g., Bretschneider or JONSWAP). Thus, a set of scaling ratios applies to specific absolute frequencies, obtained through the Doppler Shift of given encounter frequencies, and multiplication between, respectively, the scaling ratios, the encounter-wave spectrum ordinate and the frequency-derivative will make the (transformed) absolute wave spectrum available.

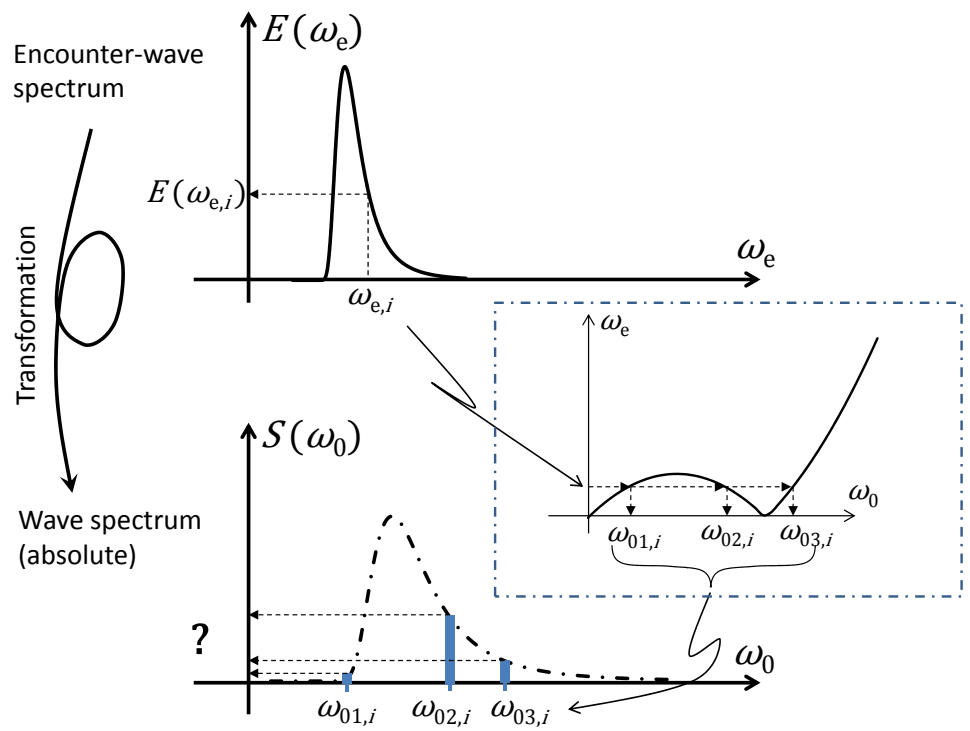

Figure 1: In following waves, it cannot be uniquely determined how the one single encountered (discrete) wave energy density, say $E\left(\omega_{e, i}\right)$, transforms into absolute domain because of the 1-to-3 relationship between frequencies. This problem occurs due to the Doppler Shift which is illustrated by the parabola inside the dashed box, showing that one encounter frequency is mapped into three absolute frequencies, whenever $\omega_{e}$ is smaller than $\frac{1}{4 \psi}$, cf. Eq. (1). 
A recent study on ship motion-based wave estimation (Nielsen et al., 2018) has shown that the transformation algorithm by Nielsen (2017) performs well in many cases related to following sea conditions. However, reports about the opposite also exist (Nielsen and Brodtkorb, 2018). Hence, having had the problem set forth, it is the purpose of this article to propose an alternative, but still practical, transformation technique which can be applied with any ship motion-based sea state estimation algorithm to transform a wave energy spectrum consistently from encounter domain to absolute domain, and at insignificant extra computational costs.

\section{Transformation to absolute domain}

In the formulation of a new transformation technique, the work should not make direct use of Eq. (4), since this was/is the solution strategy already applied by Nielsen (2017). Instead, the strategy is to take note that, despite of the fact that the individual frequency components are related through a 1-to-3 mapping (in following waves), it is possible to transform integrated parameters (such as characteristic periods, total energy content, shape parameters) if it is assumed that the absolute spectrum has a parameterised spectral shape.

In the following, the overall assumption is that the vessel speed $U$, the relative heading $\chi$ between the directions of vessel course and wave propagation, and the wave encounterspectrum $E\left(\omega_{e}\right)$ are given, equivalently said, exactly known. Secondly, it is assumed that the transformed wave spectrum $S\left(\omega_{0}\right)$ (in the absolute domain) has a spectral shape determined by the summation of two generalised JONSWAP spectra. The summation of two individual spectra has the potential to accommodate a true/generating (absolute) wave system consisting of swell and wind sea at he same time, while any single peaked generating spectrum should realise itself by fitting the two parametric spectra on top of each other. The analytical expression of the generalised JONSWAP spectrum follows from the parameterised formula (Hasselmann et al., 1973),

$$
\hat{S}\left(\omega_{0}\right)=\alpha g^{2} \frac{1}{\omega_{0}^{5}} \exp \left[-\frac{5}{4}\left(\frac{\omega_{0}}{\omega_{p}}\right)^{-4}\right] \gamma^{P} ; P=\exp \left[-\left(\frac{\frac{\omega_{0}}{\omega_{p}}-1}{\sigma \sqrt{2}}\right)^{2}\right]
$$

where the absolute frequency $\omega_{0}$ is the independent variable, while a number of (wave) parameters are conditioning variables, including the peakedness factor $\gamma$. The peak 
frequency $\omega_{p}$ is derived from the peak period $T_{p}$, while the step function $\sigma$ takes values $\sigma=0.07$ for $\omega_{0}<\omega_{p}$, and $\sigma=0.09$ for $\omega_{0}>\omega_{p}$ with $\omega_{p}=\frac{2 \pi}{T_{p}}$. Note that the constant $\alpha$ should be determined to secure conservation of energy, which requires $\int \hat{S}\left(\omega_{0}\right) d \omega_{0}=$ $\frac{1}{16} H_{s}^{2}$, where $H_{s}$ is the significant wave height. Furthermore, a Bretschneider spectrum is established by setting $\gamma=1$; otherwise, the peakedness factor takes values $1<\gamma \leq 7$. Thus, spectrum transformation results in a parameterised spectrum given by,

$$
\hat{S}\left(\omega_{0} \mid \boldsymbol{\Omega}\right)=\hat{S}_{1}\left(\omega_{0} \mid \ldots\right)+\hat{S}_{2}\left(\omega_{0} \mid \ldots\right)
$$

which is conditional on totally six parameters $\boldsymbol{\Omega}=\left\{\hat{H}_{s, 1}, \hat{T}_{p, 1}, \hat{\gamma}_{1}, \hat{H}_{s, 2}, \hat{T}_{p, 2}, \hat{\gamma}_{2}\right\}$, using the hat-symbol to indicate that the parameters relate to the transformed spectrum. Hence, the transformation problem has been converted into an optimisation problem with the objective to determine the optimum values of the six characteristic wave parameters, although it is yet unsaid what the cost function, or object function, is.

In spectral analysis, characteristic wave parameters can be calculated by the spectral moments, noting that, generally, the values of the spectral moments are (obviously!) not the same in absolute domain and in encounter domain. The n-th order spectral moment is defined by

$$
m_{n, e}=\int_{0}^{\infty} \omega_{e}^{n} E\left(\omega_{e}\right) d \omega_{e}
$$

using index 'e' to indicate that conditions in the encounter domain are considered. A variety of characteristic wave parameters can subsequently be calculated (e.g. Tucker, 1993; Krogstad et al., 1999), and examples are,

$$
\begin{array}{rlr}
H_{s, e} & =4 \sqrt{m_{0, e}} & \text { Significant wave height } \\
T_{m, e} & =2 \pi \frac{m_{0, e}}{m_{1, e}} & \text { Mean period } \\
T_{z, e}=2 \pi \sqrt{\frac{m_{0, e}}{m_{2, e}}} & \text { Zero-crossing period } \\
S_{S, e}=2 \pi \frac{H_{s, e}}{g T_{z, e}^{2}} & \text { Significant wave steepness }
\end{array}
$$

It should be clear that these parameters, in the given context, are readily available, because the encounter wave spectrum $E\left(\omega_{e}\right)$ is known. On the other hand, estimates of the particular parameters, Eqs. (8)-(11), can also be obtained by using the absolute 
spectrum, given by Eq. (6), in the calculation of the n-th order spectral moment, cf. Eq. (7). Thus, estimates of the encounter-parameters can be approximated by,

$$
m_{n, 0 \rightarrow e}=\int_{0}^{\infty} \tilde{\omega}_{e}^{n} \hat{S}\left(\omega_{0} \mid \boldsymbol{\Omega}\right) d \omega_{0}
$$

where $E\left(\omega_{e}\right) d \omega_{e}=S\left(\omega_{0}\right) d \omega_{0}$ is introduced, cf. Eq. (2), and using index ${ }_{0 \rightarrow e}$ to denote that 'encounter-condition' is obtained by using the absolute spectrum. The calculation of $m_{n, 0 \rightarrow e}$ can be done independently on the set of encounter frequencies $\omega_{e}$ (without the tilde on top), but instead any range of absolute frequencies $\omega_{0}$ can be pre-specified, whereafter $\tilde{\omega}_{e}$ is easily - and uniquely - obtained by the Doppler Shift in Eq. (1). Consequently, a cost function can be established by minimising a difference-metric obtained by comparing the actual - known - encounter-characteristic wave parameters, Eqs. (8)-(11), with corresponding estimates that are calculated by the n-th order spectral moment,

$$
m_{n, 0 \rightarrow e}=\int_{0}^{\infty}\left(\omega_{0}-\omega_{0}^{2} \frac{U}{g} \cos \chi\right)^{n} \hat{S}\left(\omega_{0} \mid \boldsymbol{\Omega}\right) d \omega_{0}
$$

for any set of pre-specified absolute frequencies, using the absolute (parameterised) wave spectrum from Eq. (6). As such, the object function is defined according to,

$$
\min _{\boldsymbol{\Omega}} \Xi^{2} \equiv \min \sum_{i}\left(\beta_{i}-\hat{\beta}_{i}\right)^{2} \quad, \quad \hat{\beta}_{i} \doteq \hat{\beta}_{i}\left(\hat{S}\left(\omega_{0} \mid \boldsymbol{\Omega}\right)\right)
$$

where the "observables" $\beta_{i}$ respectively $\hat{\beta}_{i}$ is any one of the considered characteristic wave parameters, cf. Eqs. (8)-(11); emphasising that any $\beta_{i}$ follows directly from the relevant expression in Eqs. (8)-(11) while $\hat{\beta}_{i}$ at the same time is conditioned on $\boldsymbol{\Omega}$. Altogether, the transformed spectrum is obtained by minimising the sum of the squared errors between $\beta_{i}$ and $\hat{\beta}_{i}$. Note, in the formulation herein, cf. Eqs. (8)-(11), four observables are used for the estimation of the six (unknown) wave parameters. However, if relevant, additional observables could easily been included.

\subsection{Constraints and initial values}

It is noteworthy that energy preserves (it is a requirement!) and, hence, the optimisation problem is formulated with the following constraint,

$$
\hat{H}_{s, 1}^{2}+\hat{H}_{s, 2}^{2} \equiv H_{s, e}^{2}
$$


where $\hat{H}_{s, 1}$ and $\hat{H}_{s, 2}$ are the single significant wave heights used in the parameterised absolute spectrum. Furthermore, a second type of constraint is introduced to reflect that the spectral model, i.e. the parameterised wave spectrum, should represent the expected spectral shape for a given combination of $H_{s}$ and $T_{p}$. This has been studied in detail by Torsethaugen and Haver (2004) and, based on empirical findings therein, it is suggested to impose the following additional constraint on the one (wind-dominated) wave system,

$$
\hat{\gamma}_{1}=k_{g} s_{p}^{6 / 7} ; \quad s_{p}=\frac{2 \pi}{g} \frac{\hat{H}_{s, 1}}{\hat{T}_{p, 1}^{2}} ; \quad k_{g}=35.0
$$

Like in any other optimisation, a set of initial values needs to be assigned for $\boldsymbol{\Omega}$. The initial values for the periods $\hat{T}_{p, 1}, \hat{T}_{p, 2}$ are functions of $T_{z, e}$ following the proposal made by Nielsen (2017), while the significant wave heights $\hat{H}_{s, 1}, \hat{H}_{s, 2}$ initially take equal shares of $H_{s, e}$, that is, $\hat{H}_{s, 1}=\hat{H}_{s, 2}=\frac{1}{\sqrt{2}} H_{s, e}$, and the peakedness factors are initialised by the values $\gamma_{1}=\gamma_{2}=2$.

\subsection{Other optimisation-based conversion algorithms}

It should be mentioned that the original work (Nielsen, 2017) also introduces the possibility to rely on an optimisation in the transformation of wave energy spectral densities from encounter to absolute domain. However, the fundamental solution strategy was different compared to the present work, since the cost function was established from a direct comparison of spectral densities in the two domains, leading to a non-robust and unstable solution that turned out to be sensitive to the initial guess of the wave parameters to be optimised. Likewise, a study by Montazeri et al. (2016) introduces the calculation of spectral moments as the very means for ship motion-based wave spectrum estimation. As a last note in this context, it deserves to be mentioned that attempts have also been made by Japanese researchers in the past to secure 'frequency conversions in the evaluation of wave spectra', cf. Saito and Maeda (1998), but, unfortunately, details of the work are accessible only in Japanese. Thus, any potential valuable information of this Japanese work is not clear to the author, but based on a screening of the actual paper, it appears that an optimisation strategy, like suggested in Nielsen (2017), is investigated. 


\subsection{Evaluation of performance}

In the following sections, the transformation algorithms will be tested. In Section 4, testings are made extensively on simulated data to have a solid indication of the performance levels of the old and new transformation techniques and their 'mutual consistency'. The reason to test on synthetic data is clear, as it allows complete knowledge about the processes to be studied, whereas real ocean wave conditions can be extremely difficult to accurately deal with because the truth is never exactly known. On the other hand, some means must be established to evaluate, or validate, the outcome of the transformation technique(s), in case the true generating conditions are unknown; which typically will be the case when full-scale data is studied. In the present article use will be made of full-scale data where "external" information is available by buoy measurements from a free-floating wave buoy positioned at a fixed location. Thus, testings of the transformation techniques are also made on full-scale data, cf. Section 5.

\section{Testing with numerical simulations}

In this section, the transformation techniques will be applied to transform a given encountered distribution of wave energy densities. Initially, the two algorithms are carefully compared in a study, Subsection 4.1, focused on conditions described by a unimodal sea and based on moderate forward speed. Subsequently, testings are conducted using more complex scenarios, cf. Subsections 4.2 and 4.3.

The numerical simulations will be entirely focused on following sea conditions in shortcrested (directional) waves using a plus/minus 90 degrees spreading. Realistic operational scenarios are considered and, in the context of ship motion-based sea state estimation (Nielsen et al., 2018), it means that stochastic simulations of the encountered wave spectra will be the input to the transformation techniques. The (stochastic) realisations of encounter wave spectra are obtained by simulating time histories of the encountered wave elevation process itself, where a standard simulation procedure is used (e.g. Nielsen, 2017) for the generation of the time histories. Subsequently, spectral analysis provides the "measured" wave spectrum, where for instance the WAFO toolbox (Brodtkorb et al., 2000) or the function cpsd in MATLAB ${ }^{\circledR}$ can be used to make the FFT, but noting that any standard approach can be used. The use of stochastic simulations should be 
followed by a statistical analysis/evaluation to obtain the average performance of the transformation techniques. Thus, the evaluation is based on (sub)cases that, individually, consist of totally 20 single 30 -minutes time history simulations of encountered wave elevation records.

\subsection{Case study based on a unimodal sea}

In the first case study, several subcases are studied. The subcases have as input a combination of 'generating-parameters', where the most relevant ones to study are the forward speed $U$ of the vessel, the (relative) wave heading $\chi$, and the characteristic period of the wave system, for instance the peak period $T_{p}$. Any variation in energy content, represented by significant wave height $H_{s}$, of the generating wave system is of no importance, since both the new transformation technique, Section 3, and the old one (Nielsen, 2017) preserve energy as a requirement, i.e. energy conservation is prescribed in the transformation process. The naming of the individual subcases follows a convention represented by 'U.T. $T_{p} \cdot \chi^{\prime}$, and in this way combinations are defined in accordance with Table 1 . Hence, it is understood that totally thirty $(=2 \times 3 \times 5)$ subcases exist and, for example, one specific subcase, say, B.a.IV is based on a generating-condition with $U=12$ knots, $T_{p}=8 \mathrm{~s}$, and $\chi=60 \mathrm{deg}$. Throughout, the significant wave height is $2.0 \mathrm{~m}$ but this will not be given any further attention; emphasising, and repeating, that $H_{s}$, by "default" preserves in the transformation.

Although the new and the old transformation techniques both allow for a doublepeaked (absolute) wave spectrum, the initial case study is based on numerical simulations assuming a single-peaked spectrum. Specifically, the generating point-wave spectrum is taken as a Bretschneider spectrum, cf. Eq. (5), assuming a fully developed sea.

Table 1: Naming convention of test cases: Each subcase is named 'U.T $\cdot T^{\prime}$ ' which represent a combination of the parameters of vessel speed $U$ (A or B), wave peak period $T_{p}$ (a or b or c), and relative wave heading $\chi$ (I or II or III or IV or V).

\begin{tabular}{|c|c|c|c|c|c|c|c|c|c|c|}
\hline \multirow{3}{*}{$\begin{array}{l}\text { Combination: } \\
\text { Values: }\end{array}$} & \multicolumn{2}{|c|}{$U$ [knots] } & \multicolumn{3}{|c|}{$T_{p}[\mathrm{~s}]$} & \multicolumn{5}{|c|}{$\chi$ [deg.] } \\
\hline & $\mathrm{A}$ & B & $\mathrm{a}$ & $\mathrm{b}$ & c & I & II & III & IV & $\mathrm{V}$ \\
\hline & 9 & 12 & 8 & 10 & 12 & 0 & 20 & 40 & 60 & 80 \\
\hline
\end{tabular}


Moreover, the optimisation-based results are (initially) achieved without enforcing the second constraint, cf. Eq. 16, related to the spectral shape of the (transformed) absolute wave spectrum.

The generating conditions are defined by combinations of the parameters specified in Table 1 . Hence, any wave elevation record can be simulated, using a specific parametercombination, and, subsequently, a corresponding encounter wave spectrum can be computed. It should be noted that the simulations are made with the wave spectrum truncated at $\omega_{\text {high }}=4 \pi$ (corresponding to $2 \mathrm{~Hz}$ ). An example of a particular wave elevation realisation, as measured by an advancing ship, is shown in Figure 2 that also includes the corresponding encounter-wave spectrum. With this 'sequence' at hand, the transformation to absolute domain can be made by application of the relevant transformation technique; emphasising, as mentioned previously, that the transformation takes the relative wave heading and the vessel forward speed to be (exactly) known, with values as listed in Table 1 for any given subcase. It should be noted that, by nature, ocean wave spectra are smooth in their frequency-wise density distribution. In the spectral analysis (i.e. FFT) of the wave elevation record(s), smoothing is imposed by a Parzen window applied with a $50 \%$ overlap on the full range of frequencies from the FFT.
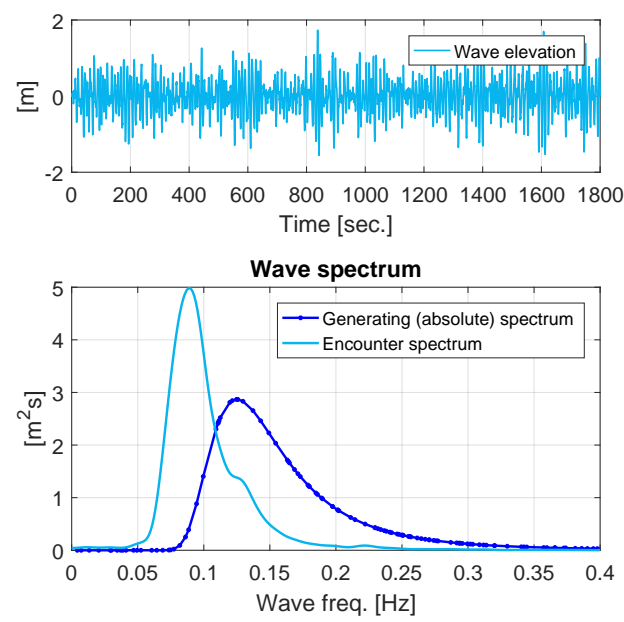

Figure 2: Top: Wave elevation record as encountered by an advancing ship $(U=9.0$ knots, $\chi=40$ deg.). Bottom: The corresponding encounter-wave spectrum, including the generating spectrum (Bretschneider: $H_{s}=2.0 \mathrm{~m}, T_{p}=8.0 \mathrm{~s}$ ). 


\subsubsection{Results and discussions}

The pair of outcomes shown in Figure 2 makes up one single realisation of encounterwave conditions, out of twenty, for one single of the thirty subcases found in Table 1. Thus, a specific (transformed) absolute wave spectrum is associated with the given encounter-wave spectrum, and for the particular realisation the result is shown in Figure 3. Note, from this point and onwards the results of the new transformation technique, Section 3, is named 'Optimisation', whereas the old technique (Nielsen, 2017) is named 'Direct'.

The entire collection of absolute wave spectra, as produced by the transformation techniques, is presented in Figures 4-6, where the left- and right-side plots present the results of $U=9$ knots and $U=12$ knots, respectively, and any pair of left- and right-side plots applies to a specific relative heading $\chi=\{0,20,40,60,80\}$ deg., for the different (generating) peak periods $T_{p}=\{8,10,12\} \mathrm{s}$ in Figures 4, 5, and 6, respectively. The general observation from the plots is that the optimisation-based transformation provides the most consistent and accurate results. The problem with the direct approach clearly manifests in some of the subcases, where energy densities are indeed "misplaced" on the transformed frequency range(s). The main concern with the optimisation-based results is the tendency that the spectral shape, in some the subcases, looks more like a "standard" JONSWAP spectrum with $\gamma \approx 2-3$, while in reality $\gamma=1$ for the generating spectrum in all the (particular) subcases, as a fully developed sea is considered.

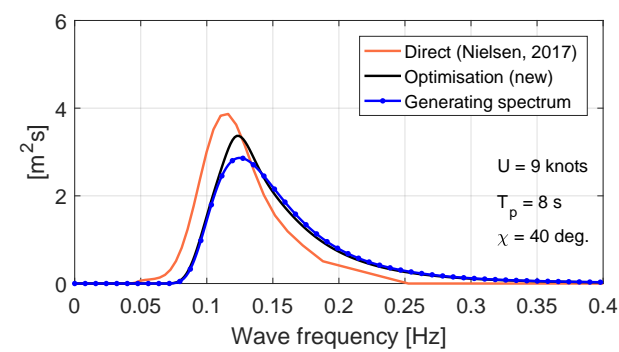

Figure 3: Transformation of encounter-wave spectrum (Figure 2) to absolute domain with results for the new optimisation-based technique (Sec. 3) and the former (more) 'direct' approach (Nielsen, 2017). The true, generating spectrum is included (Bretschneider: $H_{s}=2.0 \mathrm{~m}, T_{p}=$ $8.0 \mathrm{~s}$ ), and the conditions correspond to subcase A.a.III. 

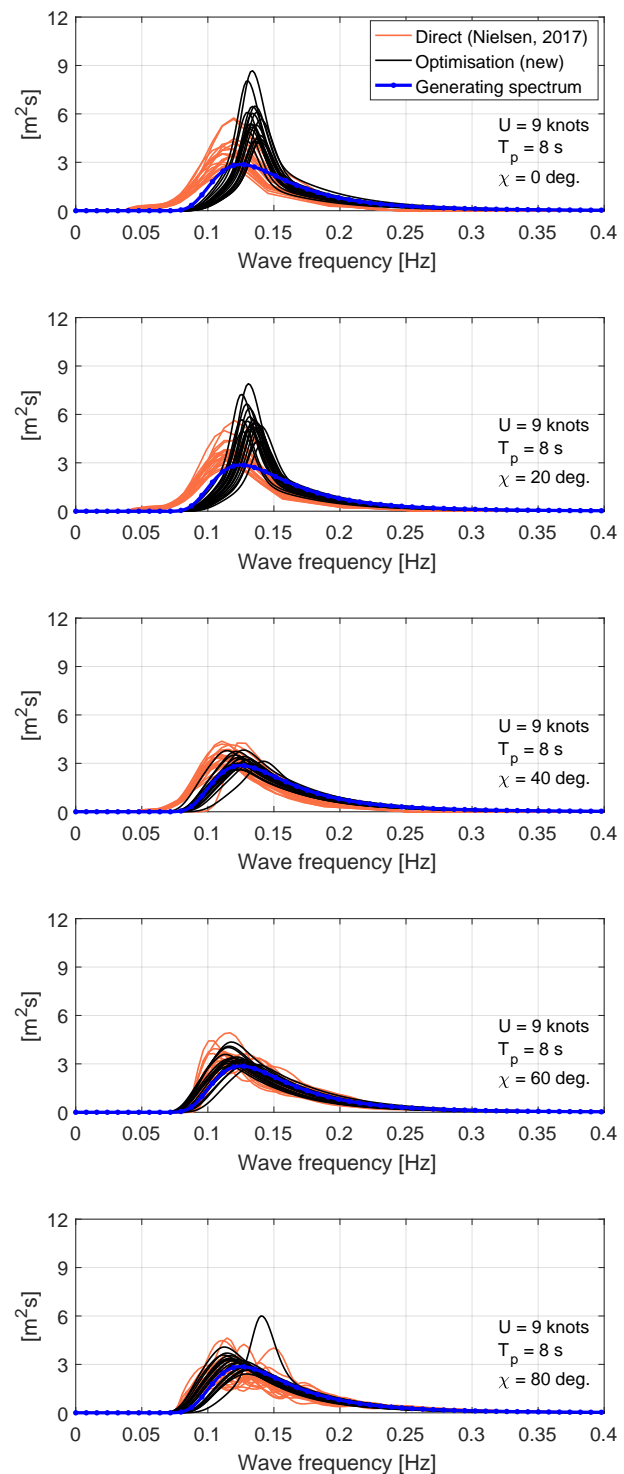
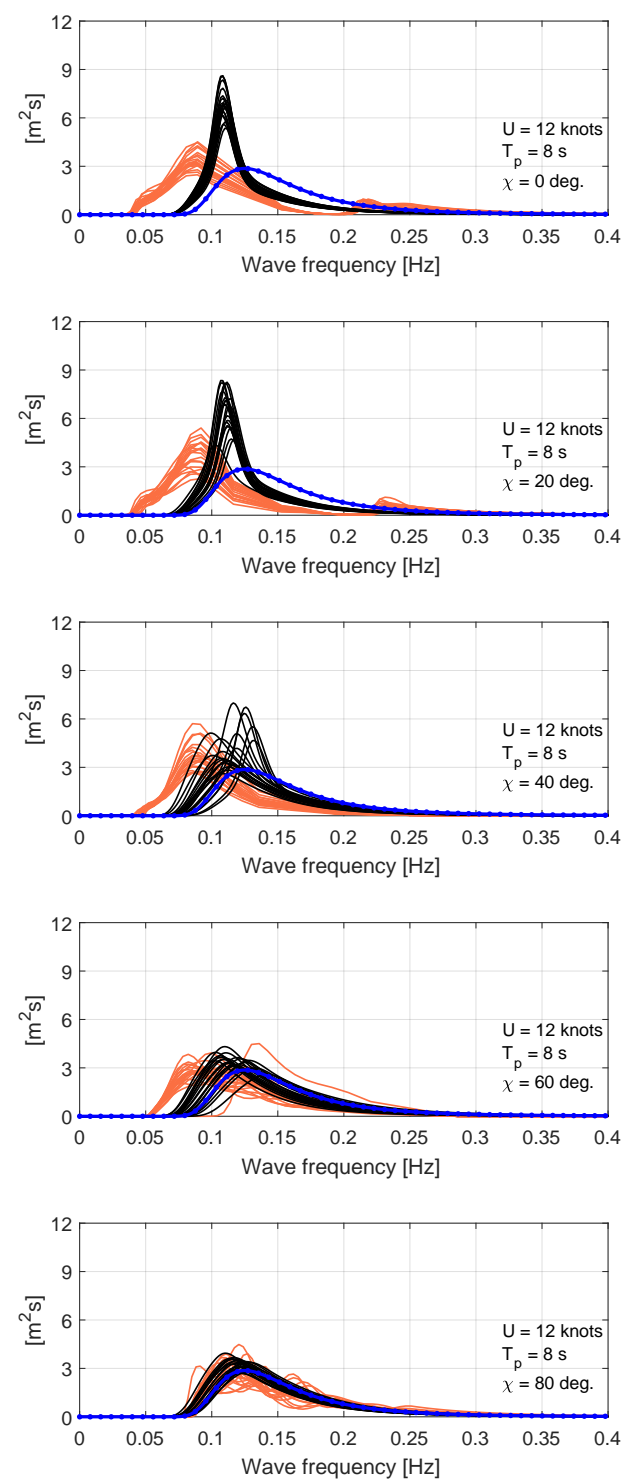

Figure 4: Absolute wave spectra of subcases A.a.I-A.a.V (left-hand side plots) and subcases B.a.I-B.a.V (right-hand side plots). 
techniques' performances, and the approach for doing this is explained below. The basis for the evaluation is the entire collection of (transformed) absolute spectra, cf. Figures 4-6. Any given absolute spectrum associates a set of (transformed) absolute wave param-
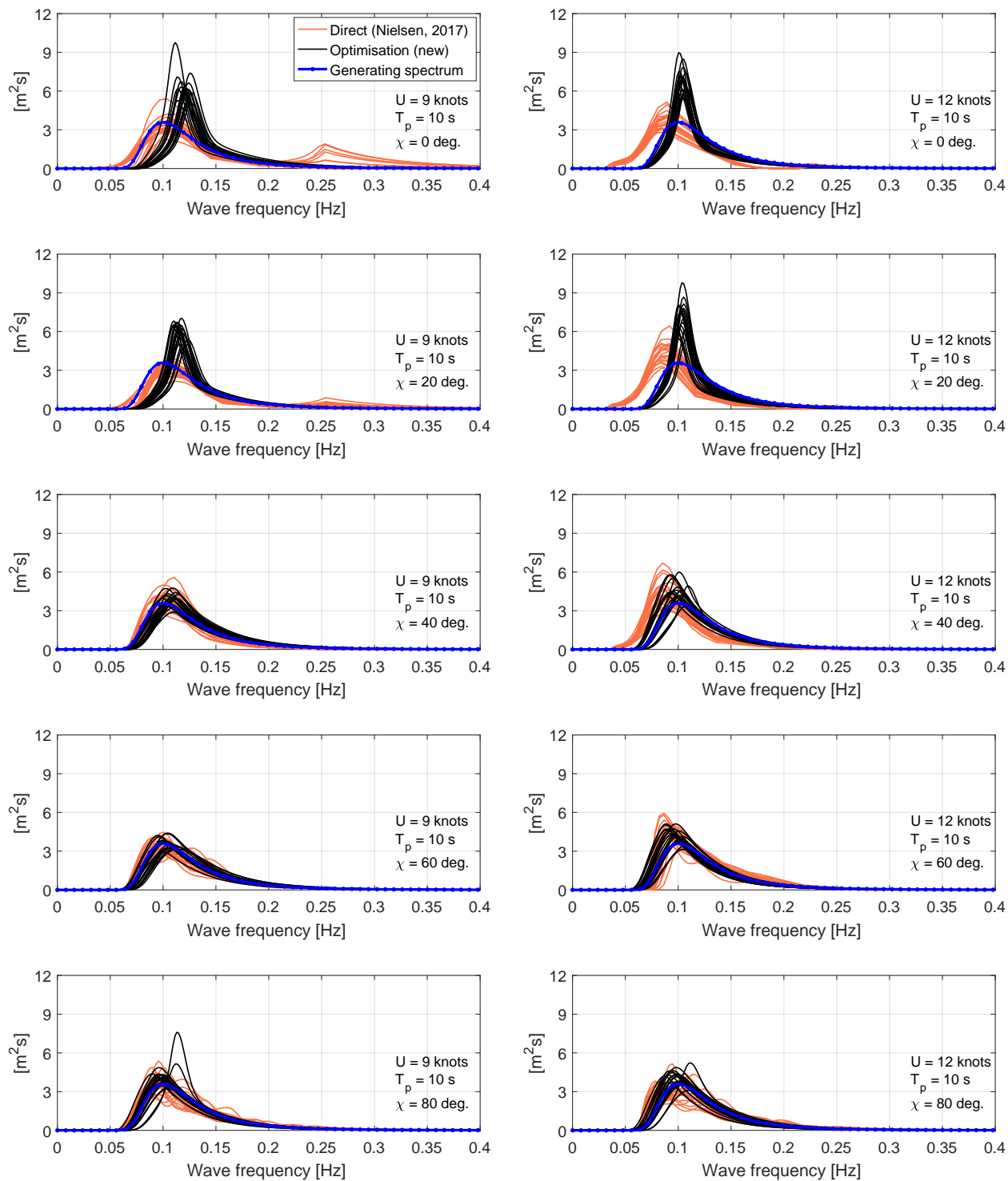

Figure 5: Absolute wave spectra of subcases A.b.I-A.b.V (left-hand side plots) and subcases B.b.I-B.b.V (right-hand side plots). 

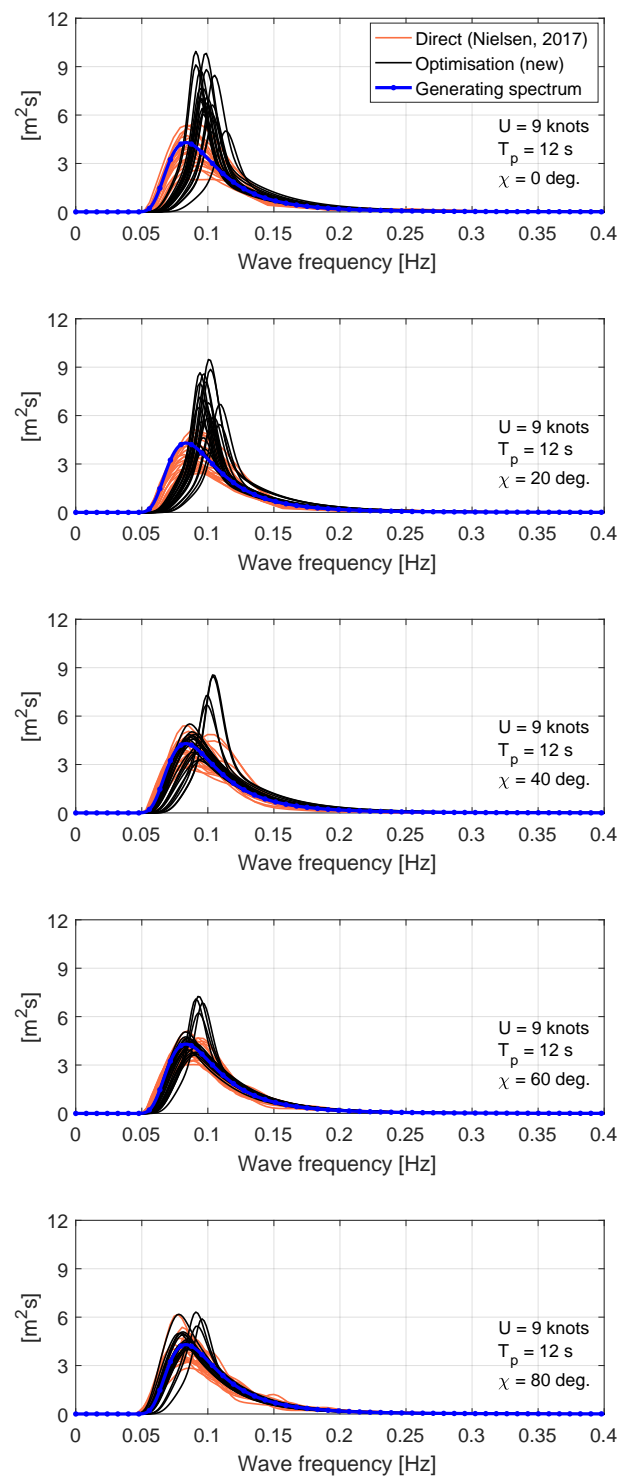
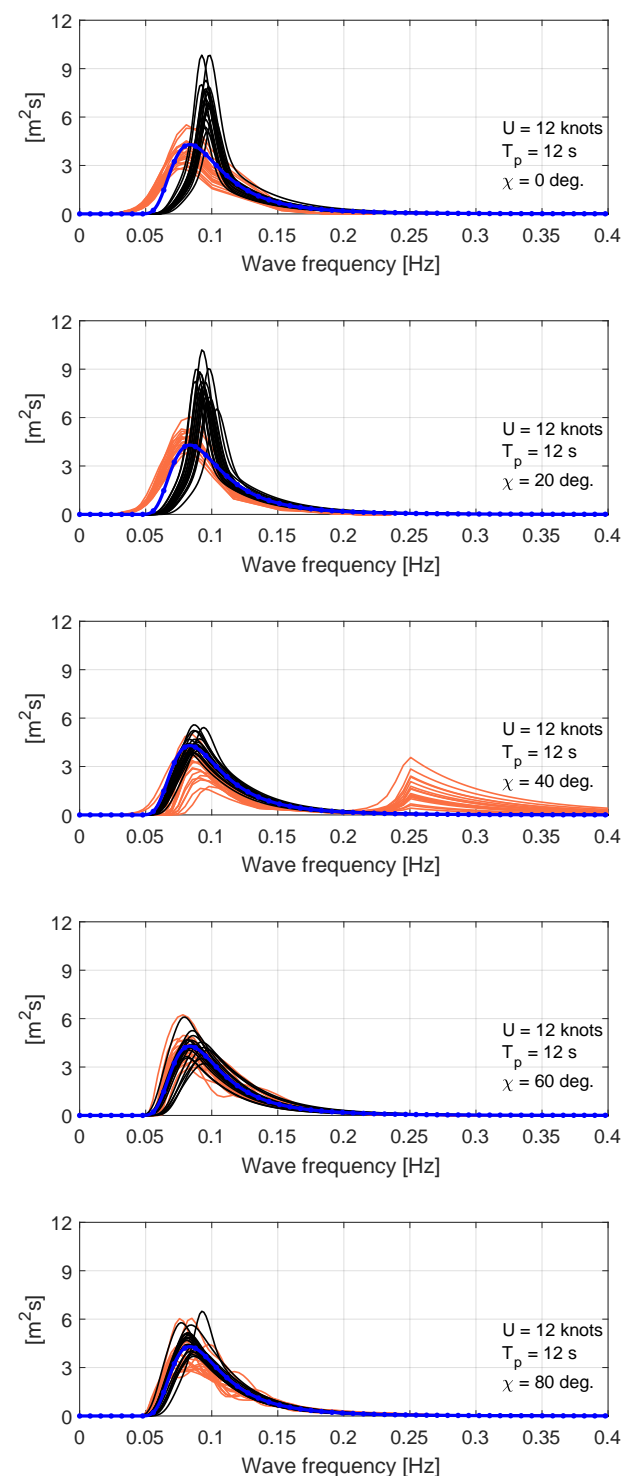

Figure 6: Absolute wave spectra of subcases A.c.I-A.c.V (left-hand side plots) and subcases B.c.I-B.c.V (right-hand side plots). 
wave spectrum is available, cf. Eqs. (8)-(11). In the end, there will be twenty of such comparisons for each of the considered subcases, cf. Table 1, and a statistical evaluation, focused on the average value of relative deviations between the 'transformed value' and the 'mutual true value', can be made. Thus, statistics are produced for the considered set of wave parameters from any one subcase's twenty outcomes, which in themselves result in plots of 'performance measures' as shown in Figure 7. In the figure, it is understood that any single point indicates the relative deviation between the transformed and the true, generating one. The particular comparison between (estimates of) transformed wave parameters to true (generating) ones is feasible only because numerical simulations are in study. In real-case scenarios, this sort of comparison is generally not possible, as it requires the (true) absolute wave spectrum to be available, which it is not in most cases.

The primary observation from Figure 7 is that the optimisation-based transformation technique consistently provides better estimates of the true absolute wave periods, where it should be kept in mind that the particular plots apply to a specific subcase, namely A.a.I, cf. Table 1 ( $U=9.0$ knots, $\chi=0 \mathrm{deg}$. for $\left.T_{p}=8.0 \mathrm{~s}\right)$. The complete statistical evaluation for each and every single subcase is observed from Figure 8 that presents the performance of the two transformation techniques when they have been applied to all
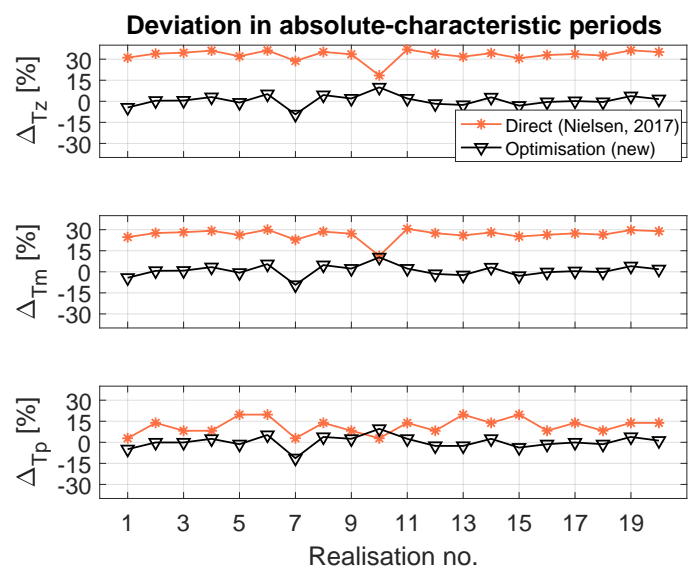

Figure 7: Comparison between transformed wave periods, from encounter to absolute domain, and corresponding generating periods of all realisations making up subcase A.a.I (cf. Table 1). Relative deviation is considered for, respectively, zero-upcrossing period (upper), mean period (middle) and peak period (lower). 

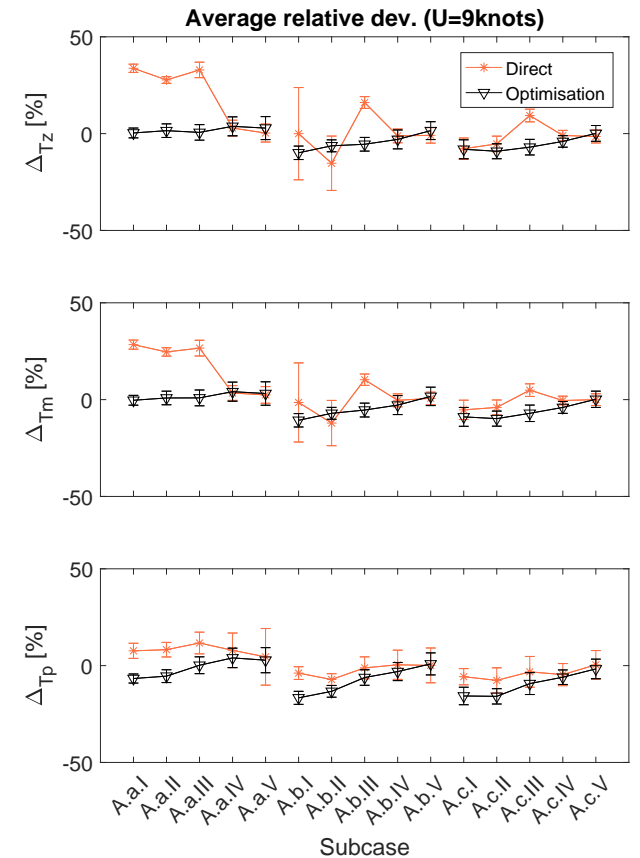
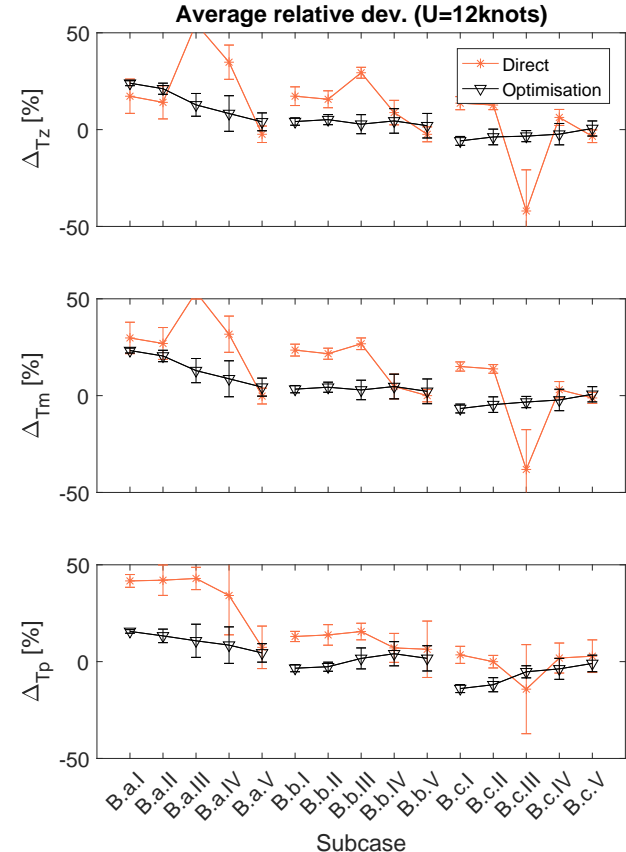

Figure 8: Statistics of relative deviation between transformed wave periods and true corresponding periods in absolute domain. Statistics are shown for the zero-upcrossing period $\left(T_{z}\right.$, top), the mean period ( $T_{m}$, middle), and peak period ( $T_{p}$, bottom). 
plots reveal that both transformation techniques provide reasonable results, although exceptions occur. For both speeds there is a similar tendency that the results of the two transformation techniques improve with account to two observations: (a) as heading becomes closer to beam sea, (b) as the wave period increases, where the findings are
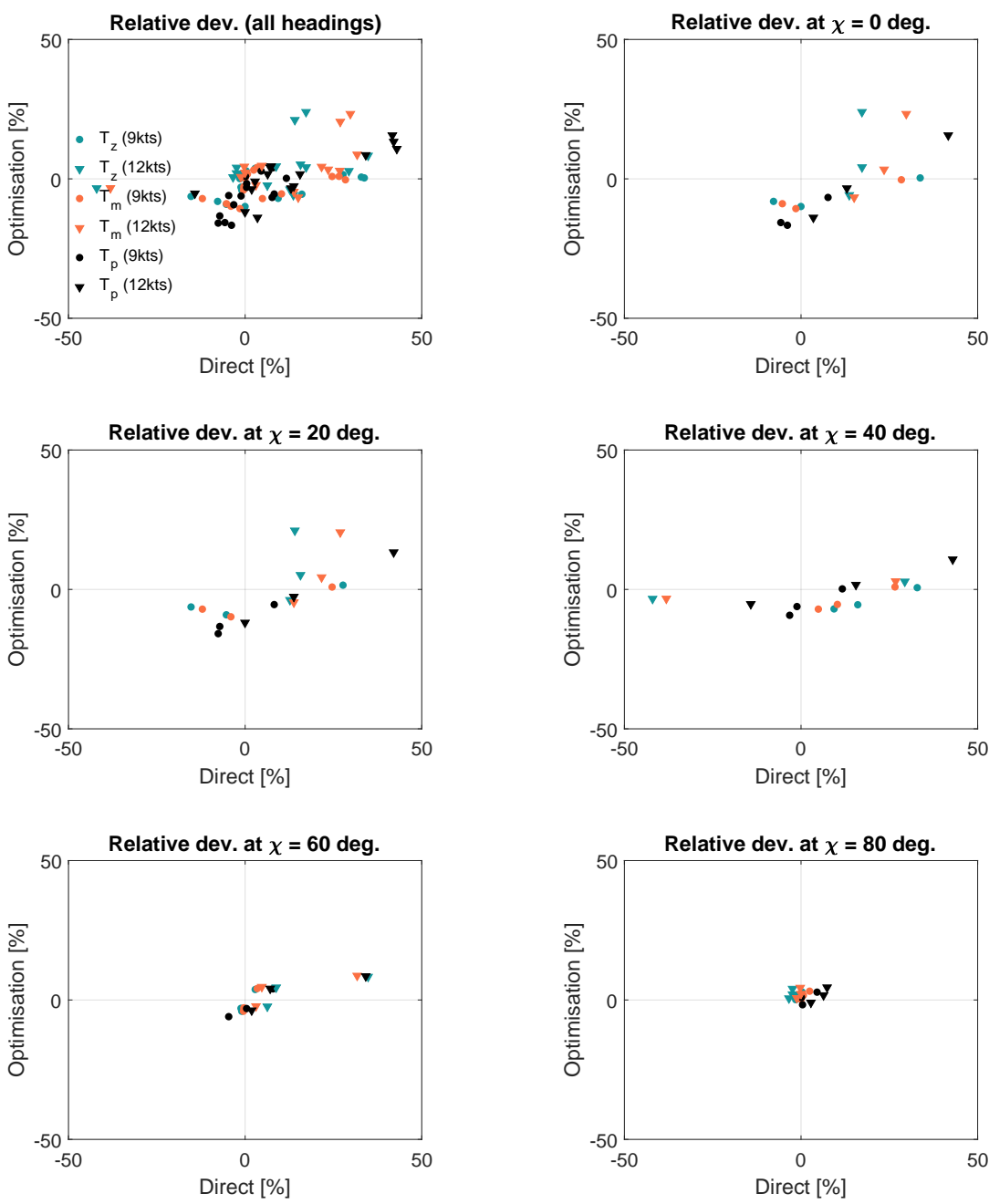

Figure 9: Comparison of statistics produced by optimisation-based technique and direct approach (Nielsen, 2017) when relative deviation between transformed wave periods and true corresponding periods in absolute domain are considered, with a "decomposition", or dependence, on heading angles. 
most notable for the direct approach. With a focus on the direct approach it can be seen that this method performs better for the lower speed ( $U=9$ knots vs. 12 knots). This observation does not show for the optimisation-based technique, where the results of the two speeds (9 knots vs. 12 knots) basically are equally good.

It may be valuable to evaluate the performance level of the transformation techniques with respect to the average accuracy considering all three characteristic periods, quantified as a whole, to have a better interpretation of a direct comparison between the two techniques. The comparison can therefore be organised more like a correlation-typeof-plot, see Figure 9. In this comparative illustration any point not located at origo represents an error relative to the exact generating value, and it should be realised that the distance from origo on the $\mathrm{y}$-axis is the error of the optimisation-based technique whereas the distance away from origo on the $\mathrm{x}$-axis is the error of the direct approach. Based on the plots in Figure 9 the most notable observations are the following: (a) the optimisation-based technique yields errors on the wave periods that are somewhat evenly distributed around zero, whereas the direct approach tends to produce wave periods which are too large; (b) the optimisation-based technique is capable to give the better results the closer the wave heading is to cases of strict following waves with $\chi=0$ deg.; (c) as the wave heading becomes closer to beam sea, the direct approach produces equally good results. It is clear that these findings can be observed also by taking a closer look at the entire set of the individual absolute spectra obtained from all the realisations, cf. Figures 4-6.

\subsubsection{Main findings}

Some overall findings and remarks based on the preceding analyses can be formulated as:

- Comprehensive testings with numerical simulations indicate that both techniques can be used to transform an encountered wave spectrum to absolute domain. However,

- the optimisation-based transformation technique is superior to the direct approach almost conclusively; emphasising that this point relates to the specific set of numerical simulations and may not be generally true. 


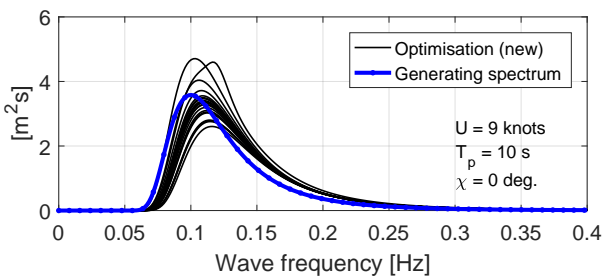
values on the higher side.

- Generally, the characteristic absolute wave parameters of the optimisation-based transformation agree with reasonable accuracy to the corresponding generating wave parameters; the exception is the spectral shape parameter $(\gamma)$ that takes

In the next subsections, additional testings are presented but a last note on the above set of results (e.g. Figures 4-6) concerns the spectral shape of the optimisation-based (transformed) spectrum. As mentioned in the last bullet point above, the value of the peakedness parameter is off, resulting in transformed spectra that look JONSWAP-like while in reality they should be Bretschneider-like. It is therefore interesting to impose the constraint in Eq. (16) that relates the spectral shape to $H_{s}$ and $T_{p}$. Figure 10 illustrates the effect of the constraint when the two subcases B.a.I and B.b.I are re-considered, cf. Table 1. It is clear that the results improve in the sense that the (transformed) absolute spectra now have a shape which agrees better with the Bretschneider spectrum; and pointing out that this finding is generally representative to the other subcases listed in Table 1. On the other hand, it calls for additional tests to study fetch-limited conditions where the JONSWAP spectrum is usually assumed to be a better match. However, this is an exercise left for the future; where the referred work by Torsethaugen and Haver (2004) should be relevant to address also in such cases.

Figure 10: The effect of the constraint, cf. Eq. (16), can be seen by re-considering subcases B.a.I and B.b.I (upper plots in Fig. 5), which were previously found to have spectral shapes similar to a JONSWAP spectrum. Indeed, the introduction of the constraint leads to a better match, and this observation is identical for all remaining cases although not shown. Note, scale on $\mathrm{y}$-axis is changed compared to Figs. 4-6. 


\subsection{Transformation at higher forward-speeds}

The subcases in the previous tests were characterised by speeds 9 and 12 knots. These values do not necessarily reflect real, in-service conditions of merchant ships. On
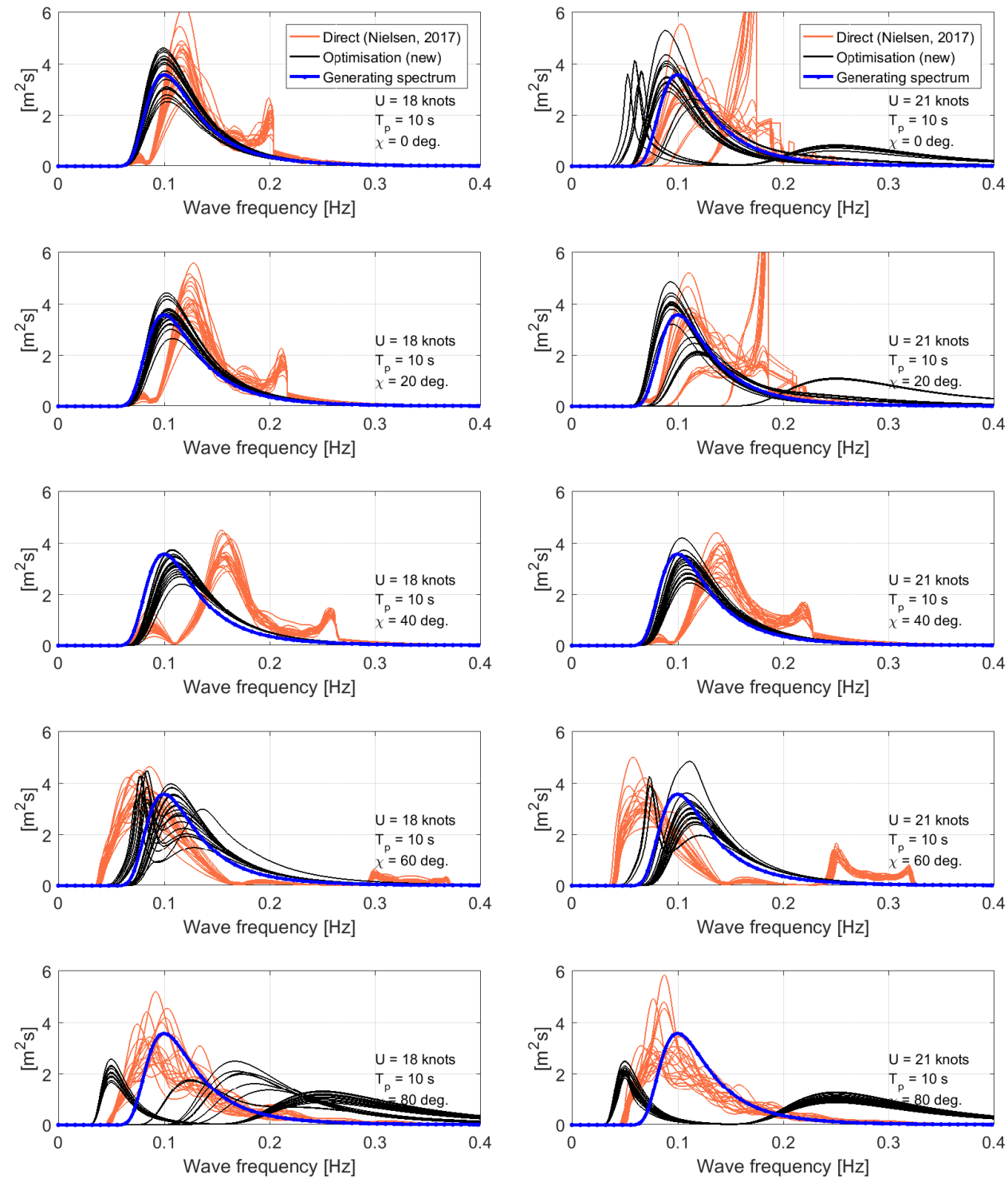

Figure 11: Absolute (transformed) wave spectra for advance speed $U=18$ knots (left-hand side plots) and $U=21$ knots (right-hand side plots). True wave peak period and wave heading appear in text within the single plots. Note, scale on y-axis is changed compared to Figs. 4-6. 
the other hand, an increase in advance speed is indeed resulting in more complex conditions due to the (stochastic) Doppler Shift, as also reported by Lindgren et al. (1999). From a theoretical viewpoint it is of interest to have efficient and accurate transformation techniques at any vessel-advance speed, but this concern is not fully applicable to practical interests. The reason is that very often the merchant ships of today rarely sail at speed levels beyond 18-20 knots, drawing attention on today's - and the future's - slow-steaming regime. And, in case a ship advances at such high speed, the severity of the wave condition can certainly not be a real issue/problem, for what reason estimates of the (absolute) wave spectrum most likely is not needed; at least not for safety reasons. Or, alternatively, the ship may spend 20-30 minutes at reduced speed (10-15 knots) to allow for (ship motion-based) wave spectrum estimation (Nielsen et al., 2018). Nonetheless, performance evaluations of the transformation algorithms have also been made on cases with higher forward-speed. Thus, transformations have been conducted at speeds 18 and 21 knots, but otherwise under similar conditions, see Table 1. The main finding from such tests is that it is possible to successfully make the transformation in many cases by the optimisation-based technique, although the performance level drops compared to the preceding subcases (9 and 12 knots). Sets of transformed spectra are shown in Figure 11 where the results for $U=18$ knots and $U=21$ knots are shown at the left-hand side and right-hand side, respectively, and showing the results just for $T_{p}=10 \mathrm{~s}$. The most important observations are: (a) The optimisation-based results are fairly consistent for $U=18$ knots and reasonable results are obtained, except for the case at $\chi=80 \mathrm{deg}$. The observations are somewhat identical for $U=21$ knots although the performance is more "obscured"; (b) The direct approach fails to transform in almost all cases for both speeds; but, importantly, it yields reasonable results at $\chi=80 \mathrm{deg}$. for both speed cases; (c) The optimisation-based approach "predict" (erroneously) a transformed spectrum with two distinct peaks for nearly all realisations related to $\chi=80$ deg. for both speeds. It is not fully understood why double-peaked (transformed) spectra manifest for $\chi=80 \mathrm{deg}$. but it should be kept in mind that the simulations are concerned with short-crested waves, based on a plus/minus 90 degrees spreading. Thus, the effect of the Doppler Shift becomes indeed (more) relevant/important for a wide range of directional wave components at higher advance speeds compared to situations at lower 
speeds. Again, it may be useful to consult the referred work about "Stochastic Doppler shift and encountered wave period distributions in Gaussian waves" (Lindgren et al., 1999, p. 514) that supports this explanation.

\subsection{Double peaked (bimodal) wave spectrum}

The final testings are made on a more complex wave scenario where a bimodal wave spectrum is considered. The test conditions are given in Table 2 and, on this basis, numerical simulations of wave elevations have been computed exactly like in the preceding; emphasising that short-crested directional waves are considered also here. From the table it is seen that totally eight $(4 \times 2)$ subcases exist, where the main differences relate to heading, studied in subcases a versus b, and speed. Moreover, two different wave conditions are studied, where cases 1 and 2 consider $T_{p}=\{15,10\}$ s for the peak periods of a swell and a wind sea system, respectively, while cases 3 and 4 consider $T_{p}=\{20,10\} \mathrm{s}$ for the peak periods of the bimodal wave system. The results are presented in Figures 12 and 13

The findings from the figures can be summarised as: (i) Overall, the best results are obtained for the lower speed $U=9$ knots (independent on the algorithm), i.e. the left-hand side plots in both figures; (ii) The optimisation-based algorithm is capable to distinctly separate the swell and wind sea peaks at $U=9$ knots for the heading $\chi=0$ deg. (iii) At the higher advance speed, the results are more obscured, and it is difficult to point out some general observations; except that for case 4a (upper right plot in Figure 13) both algorithms tend to be off, although the optimisation-based algorithm "detects" the wind sea peak correctly.

Table 2: Operational conditions and generating sea state in cases with a bimodal wave spectrum.

\begin{tabular}{lcccccccc}
\hline & & & \multicolumn{2}{c}{ Swell } & & \multicolumn{2}{c}{ Wind sea } \\
& $U[\mathrm{knots}]$ & $\beta[\mathrm{deg}$. & $H_{s}[\mathrm{~m}]$ & $T_{p}[\mathrm{~s}]$ & & $H_{s}[\mathrm{~m}]$ & $T_{p}[\mathrm{~s}]$ \\
\hline Case 1(a,b) & 9.0 & $\{0,80\}$ & 2.0 & 15.0 & & 2.0 & 10.0 \\
Case 2(a,b) & 18.0 & $\{0,80\}$ & 2.0 & 15.0 & & 3.0 & 10.0 \\
Case 3(a,b) & 9.0 & $\{0,80\}$ & 2.0 & 20.0 & & 2.0 & 10.0 \\
Case 4(a,b) & 18.0 & $\{0,80\}$ & 2.0 & 20.0 & & 2.0 & 10.0 \\
\hline
\end{tabular}



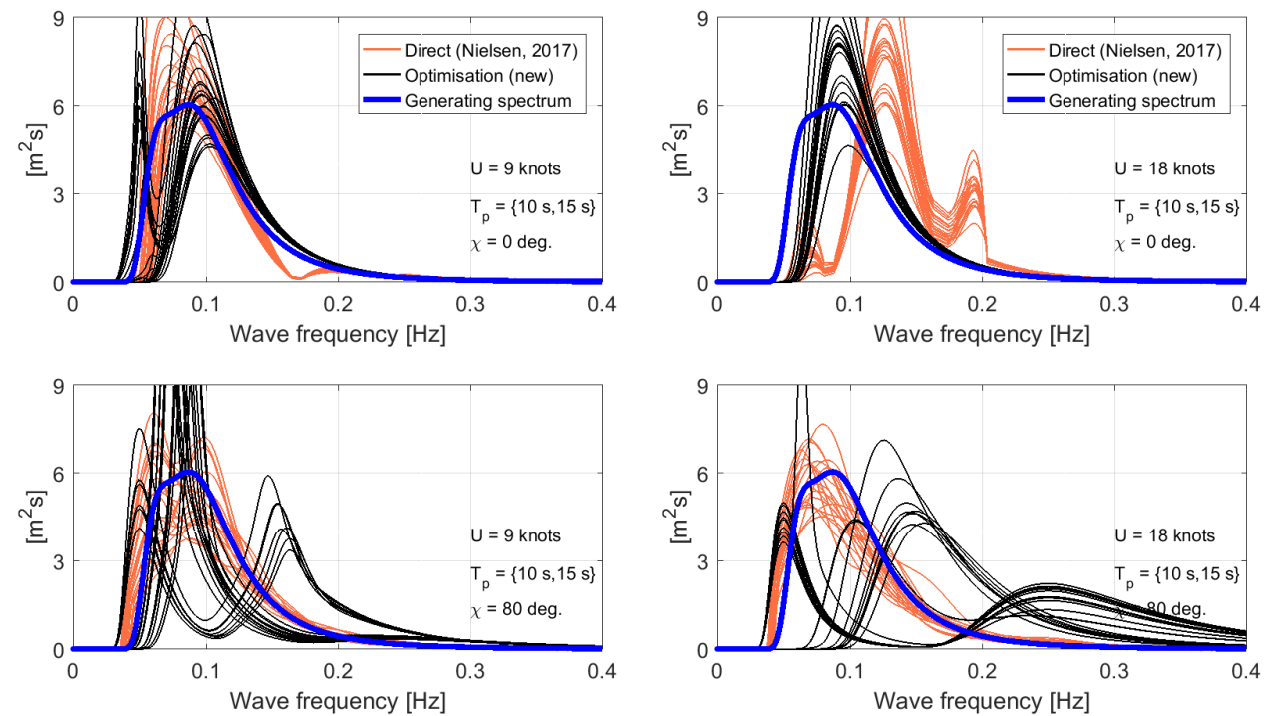

Figure 12: Comparison of transformed absolute wave spectra including the (true) generating spectrum in case of a bimodal (short-crested) sea state. Cases $1 \mathrm{a}$ and $1 \mathrm{~b}$ as the left-hand side plots, and cases $2 \mathrm{a}$ and $2 \mathrm{~b}$ as the right-hand side plots.
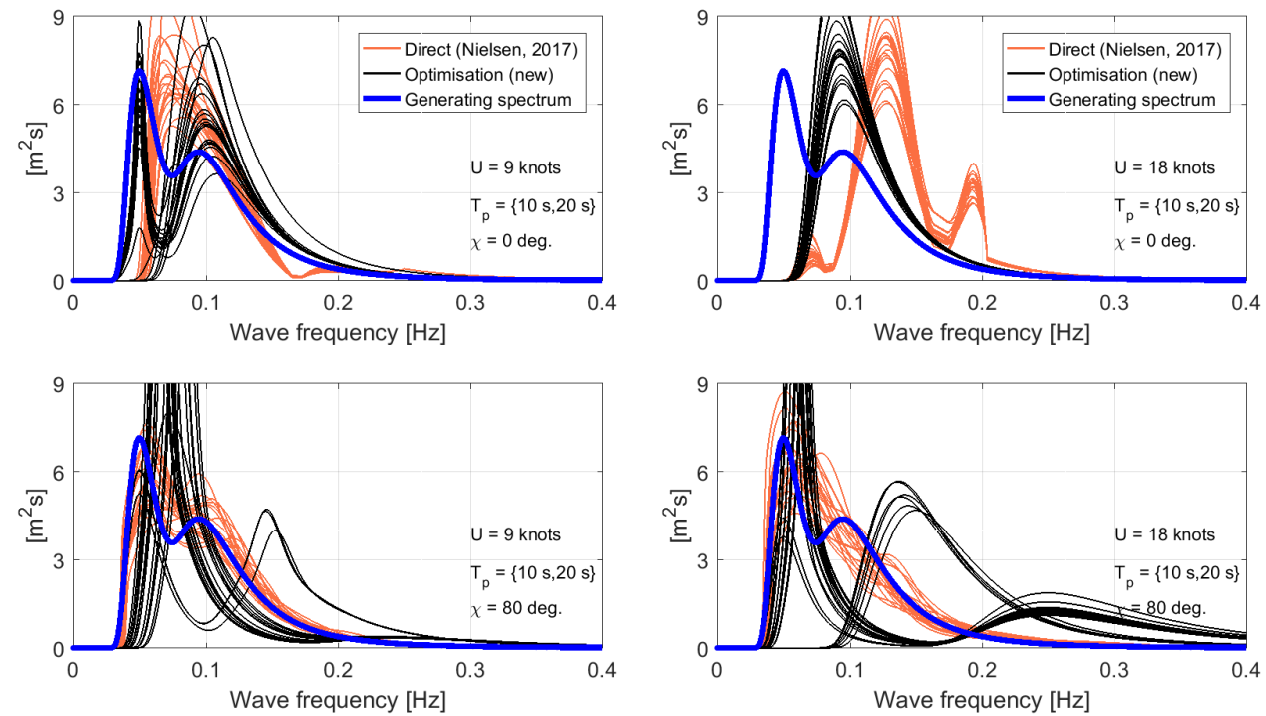

Figure 13: Comparison of transformed absolute wave spectra including the (true) generating spectrum in case of a bimodal (short-crested) sea state. Cases $3 \mathrm{a}$ and $3 \mathrm{~b}$ as the left-hand side plots, and cases $4 \mathrm{a}$ and $4 \mathrm{~b}$ as the right-hand side plots. 


\section{Testing with full-scale experimental data}

\subsection{Measurements from sea trials}

Experimental data has been collected during sea trials with a smaller research vessel, $R / V$ Gunnerus owned and operated by the Norwegian University of Science and Technology. The specific vessel is characterised by main dimensions: Length $L_{p p}=28.9 \mathrm{~m}$, breadth $B=9.6 \mathrm{~m}$, draught $T=2.7 \mathrm{~m}$, and block coefficient $C_{B}=0.56$. A more detailed description of the sea trials, and the studied vessel, is given by Steen et al. (2016), and
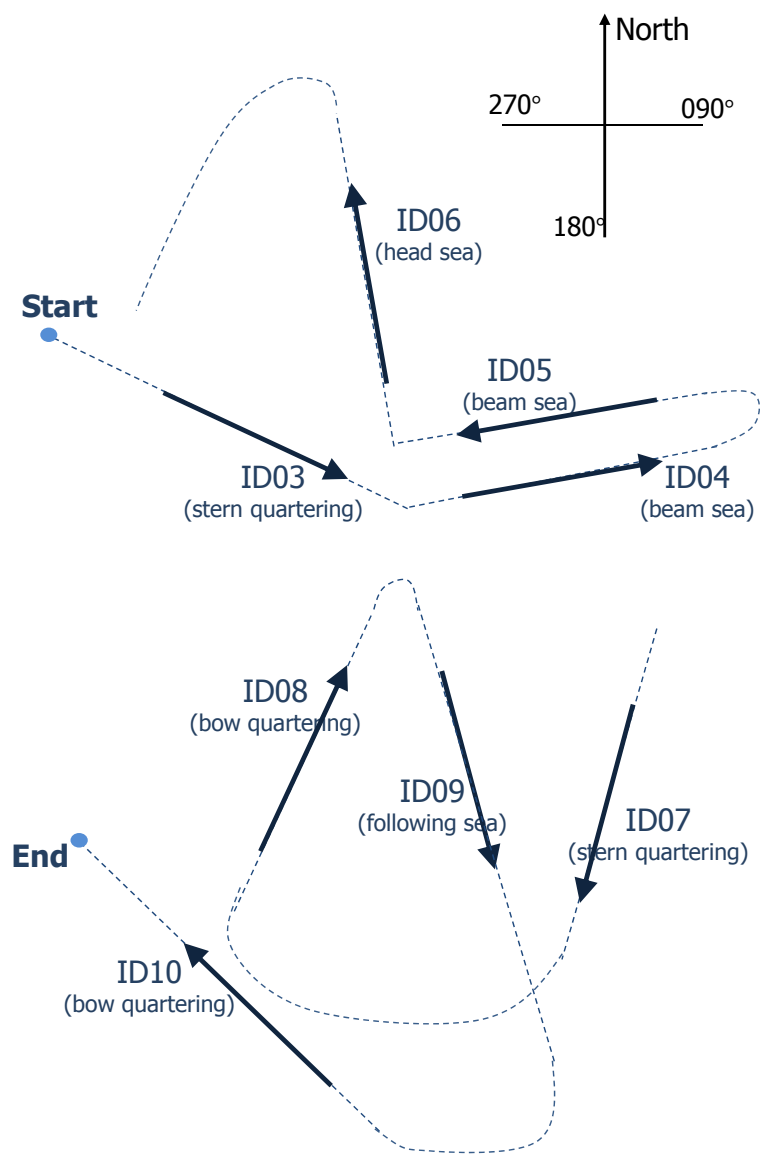

Figure 14: Vessel run paths during sea trials; IDs 03-06 at top and IDs 07-10 at bottom with a very short transition time in between all runs. The (visually) observed wave heading is included in parentheses; noting that the observed wave direction was nearly the same during all runs, with waves coming from North North-East. From Nielsen and Brodtkorb (2018). 

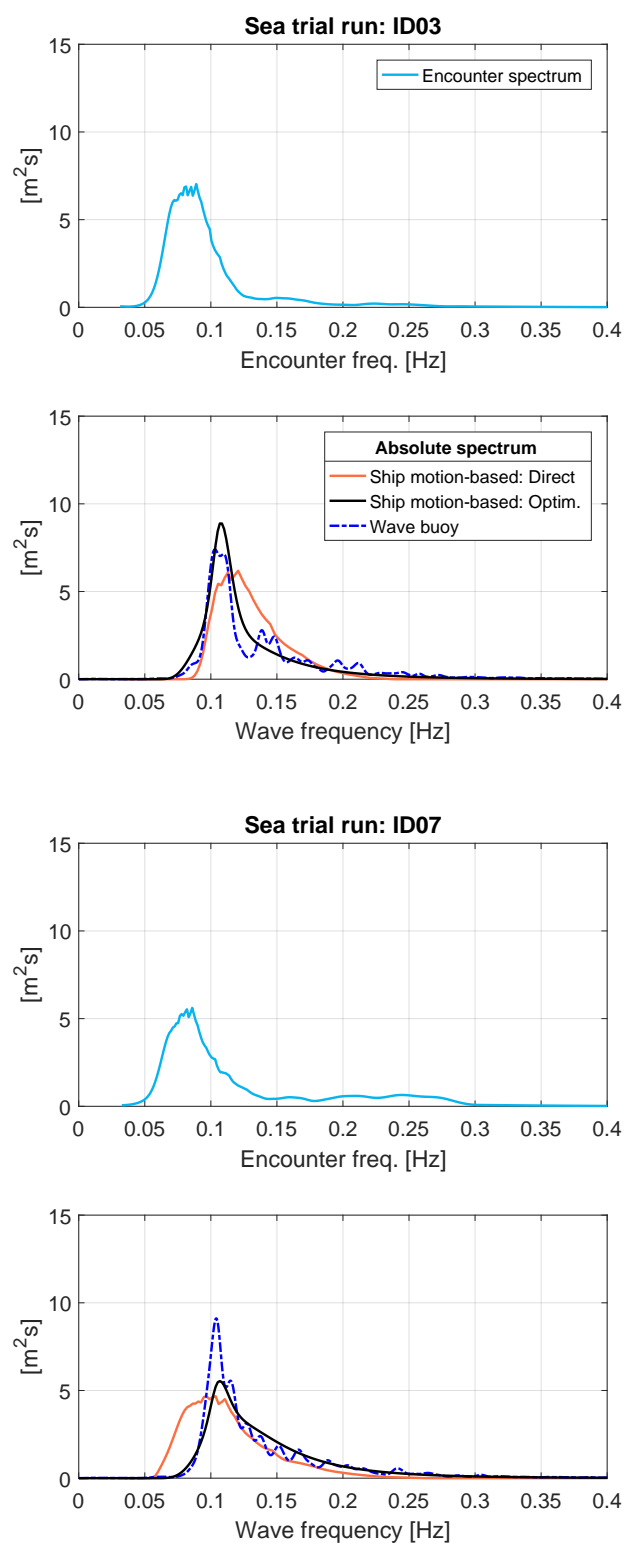
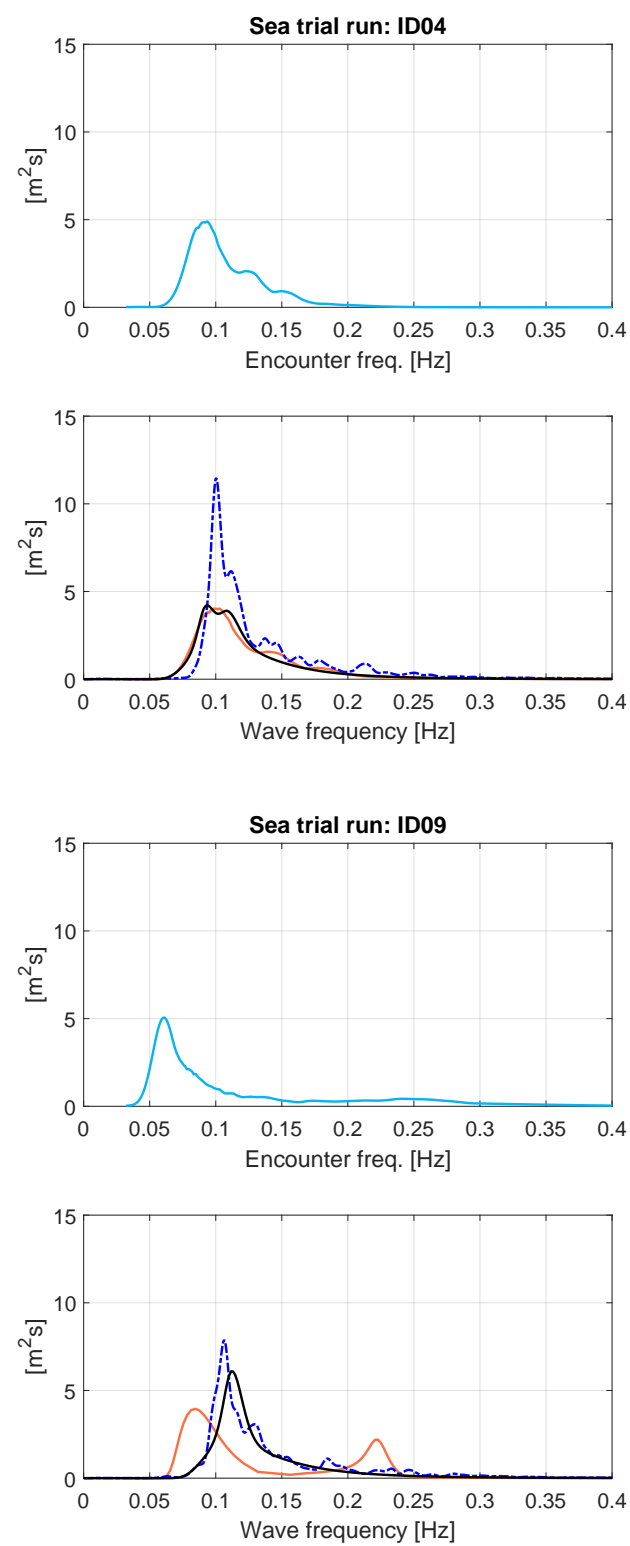

Figure 15: Pairs of encounter wave spectrum and corresponding absolute wave spectrum as obtained from experimental sea trials data (Nielsen and Brodtkorb, 2018). 
illustrated in Figure 14, where it is noted that the analysed motion recordings have been obtained on the single straight-line paths. On each path, course and engine power were held constant to secure stationary conditions, and the durations of the runs were 25-30 minutes. The detailed outcome of the ship motion-based wave spectrum estimation will not be presented herein. Instead reference is given to Nielsen et al. (2018) and Nielsen and Brodtkorb (2018), pointing out that the outcome of the ship motion-based estimation includes the encounter wave spectrum. As part of the sea trials data, information was also available about the absolute wave spectrum, which was measured continuously by a free-floating wave buoy (Datawell BV) deployed at a fixed position in the test area.

\subsection{Performance of transformation algorithms}

The two transformation techniques have been applied to transform the measured encountered-wave energy density distributions to corresponding absolute wave spectra.

The run pattern, cf. Figure 14, of the sea trials data shows that four particular run paths correspond to following sea conditions. The runs are ID03, ID04, ID07, and ID09, and it is noteworthy that the advance speed of the vessel has been reported as 10-12 knots in the specific cases. The outcomes of the analyses are shown in Figure 15, where the pairs of upper and lower plots show the estimated encounter-wave spectrum and, respectively, the (transformed) spectrum estimate in absolute domain together with a corresponding estimate by a free-floating wave buoy.

Inspection of the plots in Figure 15 reveals that the two transformation techniques give fairly consistent results in three of the cases, but in the one case, ID09, there is an inconsistency. The comparison with corresponding wave spectrum measurements by a free-floating wave buoy favours the optimisation-based transformation technique. Thus, it is evident that the absolute wave spectra produced by the optimisation-based technique are indeed consistent with corresponding estimates by the wave buoy.

\section{Summary and suggested future work}

In the context of ship motion-based wave spectrum estimation, one well-performing methodology (Nielsen et al., 2018) requires a means to transform an encountered distribution of wave energy densities to absolute domain. In this article, an optimisation-based 
technique to transform encounter-wave spectra to absolute domain has been proposed.

Comprehensive testings with simulated data, based on a unimodal wave spectrum, indicated a high level of performance of the specific transformation technique. Investigations of the performance were conducted at different advance speeds, in the order 9-21 knots, and at various 'generating conditions' of the wave system. Along the investigations, comparisons were made to an existing transformation technique (Nielsen, 2017). It became clear from the studies of simulated data that the (new) optimisation-based technique led to more consistent and accurate absolute wave spectra, and the same finding was made in a study using experimental full-scale sea trials data. Testings at the higher advance speeds (18-21 knots), for numerically simulated data, indicated a decrease in the general performance level of the transformation algorithms. Additional tests, based on numerical simulations using a bimodal wave spectrum, confirmed that that the transformation algorithm(s) perform satisfactorily at lower speeds, while the performance drops at higher speeds (18 knots). In this connection, it needs, however, to be realised that, if the purpose of transformation relates to shipboard wave spectrum estimation (Nielsen et al., 2018), the particular vessel should be able to reduce speed in a shorter period of time (20-30 minutes) to have consistent and reliable estimates of the - absolute - wave spectrum; pointing out also that very often the merchant ships of today rarely sail at speed levels beyond 18-20 knots, drawing attention on today's - and the future's - slow-steaming regime.

It is suggested that future work addresses proposals on the following list:

- Most of the analyses in this study were focused on numerically simulated data, while less focus was given to (full-scale) experimental data. Obviously, the transformation algorithm(s) should be tested more carefully with in-service and/or sea trial data.

- From the analyses it was observed, in some cases, that the optimisation-based algorithm failed to transform accurately, while, for the same cases, the direct algorithm (Nielsen, 2017) performed satisfactorily. Obviously, it should therefore be interesting to combine the two algorithms. Note, if such a combined algorithm were available, the use of a parameterised wave spectrum would also be relaxed, since the direct algorithm (Nielsen, 2017) is semi-nonparametric. 
- The study of simulated data, or experimental data with external wave information available, e.g. by buoy measurements, allows direct validation of the transformation technique by comparing its outcome to the true "generating" conditions. However, in practice, estimates of the generating wave conditions will typically not be available and, hence, some other way to validate the (transformed) absolute wave spectrum should be developed.

- The particular transformation algorithms are (usually) applied for shipboard sea state estimation based on measured vessel motions. It should therefore be interesting to apply a given (transformed) absolute wave spectrum to make 'repeated response calculations', i.e. to simulate the real motion measurements. Thus, if the measurements can be successfully simulated by using the transformed absolute wave spectrum, in a given case, it is highly likely that the specific transformed spectrum is correct.

\section{Acknowledgement}

Thanks are directed to two anonymous reviewers for constructive feedback and suggestions. This work has been supported by the Research Council of Norway through the Centres of Excellence funding scheme, Project number 223254-AMOS.

\section{References}

Beck, R., Cummins, W., Dalzell, J., Mandel, P., Webster, W., 1989. Vol. III: Motions in Waves and Controllability. In: Lewis, E. (Ed.), Principles of Naval Architecture, Second Revision. SNAME, pp. $1-188$.

Bhattacharyya, R., 1978. Dynamics of Marine Vehicles. John Wiley \& Sons.

Brodtkorb, A. H., Nielsen, U. D., Sørensen, A. J., 2018. Sea State Estimation Using Vessel Response in Dynamic Positioning. Applied Ocean Research 70, 76-86.

Brodtkorb, P. A., Johannesson, P., Lindgren, G., Rychlik, I., Ryden, J., Sjö, E., Sköld, M., August 2000. WAFO - a Matlab Toolbox for Analysis of Random Waves and Loads. (The WAFO package can be downloaded freely on the internet.).

Hasselmann, K., Barnett, T., Bouws, E., Carlson, H., Cartwright, D., Enke, K., Ewing, J., Gienapp, H. Hasselmann, D., Kruseman, P., Meerburg, A., Mller, P., Olbers, D., Richter, K., Sell, W., Walden, H., 1973. Measurements of wind-wave growth and swell decay during the Joint North Sea Wave 
Project (JONSWAP). Ergnzungsheft Reihe A(8) - 12, Deutches Hydrographisches Institut, Hamburg, Germany.

Iseki, T., Ohtsu, K., 2000. Bayesian estimation of directional wave spectra based on ship motions. Control Engineering Practice 8, 215-219.

Journée, J., Massie, W., January 2001. Offshore Hydromechanics. Lecture notes in course offered at TU Delft.

Krogstad, H., Wolf, J., Thompson, S., Wyatt, L., 1999. Methods for intercomparison of wave measurements. Coastal Engineering 37, 235-257.

Lewandowski, E. M., 2004. The Dynamics of Marine Craft: Maneuvering and Seakeeping. World Scientific.

Lindgren, G., Rychlik, I., Prevosto, M., 1999. Stochastic Doppler shift and encountered wave period distributions in Gaussian waves. Ocean Engineering 26, 507-518.

Montazeri, N., Nielsen, U. D., Jensen, J. J., 2016. Estimation of wind sea and swell using shipboard measurements - A refined parametric modelling approach. Applied Ocean Research 54, 73-86.

Nielsen, U. D., 2006. Estimations of on-site directional wave spectra from measured ship responses. Marine Structures 19, 33-69.

Nielsen, U. D., 2008. Introducing two hyperparameters in Bayesian estimation of wave spectra. Probabilistic Engineering Mechanics 23, 84-94.

Nielsen, U. D., 2017. Transformation of a wave energy spectrum from encounter to absolute domain when observing from an advancing ship. Applied Ocean Research 69, 160-172.

Nielsen, U. D., Brodtkorb, A. H., 2018. Ship motion-based wave estimation using a spectral residualcalculation. In: Proc. of MTS/IEEE OCEANS18. Kobe, Japan.

Nielsen, U. D., Brodtkorb, A. H., Sørensen, A. J., 2018. A brute-force spectral approach for wave estimation using measured vessel motions. Marine Structures 60, 101-121.

Pascoal, R., Perera, L. P., Soares, C. G., 2017. Estimation of Directional Sea Spectra from Ship Motions in Sea Trials. Ocean Engineering 132, 126-137.

Pascoal, R., Soares, C. G., Sørensen, A. J., 2007. Ocean Wave Spectral Estimation Using Vessel Wave Frequency Motions. Journal of Offshore Mechanics and Arctic Engineering 129, 90-96.

Price, W. G., Bishop, R. E. D., 1974. Probalistic Theory of Ship Dynamics. Chapman and Hall.

Saito, K., Maeda, K., 1998. An Estimation of Wave Characteristics Based on Measured Ship Motions Frequency Conversions in the Evaluation of Wave Spectra (in Japanese). J. of the Society of Naval Architects of Japan 184, 159-165.

Steen, S., Selvik, Ø., Hassani, V., 2016. Experience with rim-driven azimuthing thrusters on the research ship Gunnerus. In: Proc. of High-Performance Marine Vessels. Cortona, Italy.

Tannuri, E. A., Sparano, J. V., Simos, A. N., Cruz, J. J. D., 2003. Estimating directional wave spectrum based on stationary ship motion measurements. Applied Ocean Research 25, 243-261.

Torsethaugen, K., Haver, S., 2004. Simplified double peak spectral model for ocean waves. In: Proc. 14th ISOPE. Toulon, France.

Tucker, M., 1993. Recommended standard for wave data sampling and near-real-time processing. Ocean 
Engineering 20, 459-474. 